\title{
Treat 2019 novel coronavirus (COVID-19) with IL-6 inhibitor: Are we already that far?
}

\author{
Yuanyuan Zhang, Yanyan Zhong, Lin Pan, Jing Dong* \\ Department of Rheumatology and Clinical Immunology, The Affiliated Hospital of Qingdao University, Qingdao, Shandong, China.
}

\begin{abstract}
SUMMARY The world is in the midst of the coronavirus disease 2019 (COVID-19) pandemic. Interleukin 6 (IL-6) inhibitor (tocilizumab) had been suggested for the treatment of acute respiratory distress syndrome (ARDS) patients based on the concept of "cytokine storm" in COVID-19. However, we still lack reliable studies to verify "cytokine storm" in COVID-19 pneumonia. Furthermore, IL-6 inhibitor has potential hazards of inducing infectious diseases. The efficacy of IL-6 monoclonal antibody-directed therapy remains to be fully evaluated.
\end{abstract}

Keywords coronavirus, cytokine storm, pneumonia, tocilizumab

The pandemic of coronavirus disease 2019 (COVID-19) is a highly contagious respiratory disease resulting from a life-threatening novel coronavirus, SARS-CoV-2. Since its outbreak in Wuhan, China in December 2019, it has now spread to 213 countries, areas or territories, causing a severe public health burden (1). There is an urgent need for effective treatment. Current focus has been on the development of novel therapeutics, including antiviral drugs and vaccines. Remdesivir, which has been recognized as a promising antiviral drug against a wide array of RNA virus infections, has been revealed to be highly effective in the control of COVID-19 infection in vitro (2). A compassionate-use cohort suggests that remdesivir may have clinical benefit in patients with severe COVID-19 (3). However, there are still no randomized, placebo-controlled trials of antiviral therapy for COVID-19.

The most common clinical presentation of COVID-19 infection is fever, fatigue, and dry cough. Notably, $11-53 \%$ of pneumonia patients developed acute respiratory distress syndrome (ARDS) in different cohorts, and respiratory failure from ARDS is the leading cause of mortality (4-7). The pathophysiology of COVID-19 has not been completely understood. In other respiratory infections like influenza and Middle East respiratory syndrome coronavirus (MERS-CoV), severe "cytokine storm", with markedly higher levels of proinflammatory cytokines including interferons (IFNs), tumor necrosis factors (TNFs), interleukins (ILs), and chemokines, has been reported in severe hospitalized patients $(8,9)$. Following previous experiences in severe acute respiratory syndrome (SARS) infected subjects, the Chinese National Health Commission (NHC) has recommended steroids as standard therapy for severe COVID-19 pneumonia although their role remains controversial. A new approach of targeting "cytokine storm" in severe COVID-19 patients emerged from clinical experiences in China. Recently, interleukin 6 receptor (IL-6R) monoclonal antibody (tocilizumab)directed COVID-19 therapy has been used in a clinical trial in China (No.ChiCTR2000029765), and it has been incorporated into COVID-19 management guidelines generated in China based on the concept of "cytokine storm" in COVID-19 pneumonia (10). However, the role of interleukin 6 (IL-6) in COVID-19 is still unknown, and the efficacy of IL-6 monoclonal antibody-directed therapy remains to be fully evaluated.

One study that was conducted in the early epidemic period of COVID-19, reported that patients requiring ICU admission had higher concentrations of proinflammatory cytokines (GCSF, IP10, MCP1, MIP1A, and TNF $\alpha$ ) than did those not requiring ICU admission, suggesting that the cytokine storm was associated with disease severity. However, they also found increased secretion of T-helper-2 (Th2) cytokines (e.g. IL-4 and IL-10) that suppress inflammation, which couldn't be elucidated well (11). Some recent studies also show that IL-6 level increased in severe patients $(7,12,13)$. Serum viral load detected by RTPCR is closely correlated with IL-6 level in critically ill COVID-19 patients (14). These reports provide valuable information on the immunopathology of COVID-19. However, it is important to bear in mind that it is hard to make a conclusion of "cytokine storm" based solely 
on elevated IL-6 level. Importantly, PCR positivity does not necessarily indicate viable virus, and additional data are needed to better understand the infectious period of COVID-19 and implications for treatment and infection control. A preprint article reported high levels of three cytokines (CXCL10, CCL7 and IL-1 receptor antagonist), rather than IL-6, were associated with increased viral load, loss of lung function, lung injury and a fatal outcome in clinically moderate and severe COVID-19 patients (15). Furthermore, high levels of a particular cytokine that strongly correlates with disease activity do not necessarily constitute causality. It is presently unclear if elevated IL-6 levels are detrimental or beneficial in COVID-19 pneumonia. In experimental model systems, IL- 6 can either suppress or facilitate viral replication (16), so studies on COVID-19 are urgently needed. Timing of anti-IL-6R, if too early might adversely affect viral clearance, which needs to be assessed in trials (17). Some scientists may refer to the postmortem pathology findings of COVID-19 as one of evidence for cytokine storm (18). In this study, they reported there was an increased concentration of highly proinflammatory CCR4+CCR6+ Th-17 in CD4 $\mathrm{T}$ cells which implied that overactivation of $\mathrm{T}$ cells accounts for the severe immune injury. However, they did not mention the time point when the peripheral blood was prepared for flow cytometric analysis. If the blood was taken right before the patient's death rather than before the onset of ARDS, using the result to account for immune injury would be unpersuasive. In conclusion, the available studies may be helpful for encouraging further, more in-depth research, but they should not be regarded as definitive evidence of tocilizumab therapy.

Indeed, "cytokine storm" had been reported in many diseases including infections and rheumatic diseases. Macrophage activation syndrome (MAS), for example, refers to acute overwhelming inflammation caused by a "cytokine storm" (19). The fact that biologic agents neutralizing IL-6 and IL-1 are highly effective treatments for sJIA - a rheumatic disease strongly associated with MAS - raised hopes that the same strategies would be successful to prevent cytokine storm in inflammation. However, we should be very cautious about these biologics since they have potential hazards of inducing infectious diseases.

In conclusion, we now have a better understanding of the IL-6 change and cytokine storm in COVID-19 pneumonia, but more data are needed on treatment options that improve survival.

\section{References}

1. World Health Organization. Coronavirus disease (COVID-19) outbreak. https://www.who.int (accessed April 18, 2020).

2. Wang M, Cao R, Zhang L, Yang X, Liu J, Xu M, Shi Z, Hu Z, Zhong W, Xiao G. Remdesivir and chloroquine effectively inhibit the recently emerged novel coronavirus (2019-nCoV) in vitro. Cell Res. 2020; 30:269-271.

3. Grein J, Ohmagari N, Shin D, et al. Compassionate use of remdesivir for patients with severe Covid-19. N Engl J Med. 2020; doi: 10.1056/NEJMoa2007016.

4. Chen N, Zhou M, Dong X, Qu J, Gong F, Han Y, Qiu Y, Wang J, Liu Y, Wei Y, Xia J, Yu T, Zhang X, Zhang E. Epidemiological and clinical characteristics of 99 cases of 2019 novel coronavirus pneumonia in Wuhan, China: A descriptive study. Lancet. 2020; 395:507-513.

5. Zhou F, Yu T, Du R, et al. Clinical course and risk factors for mortality of adult inpatients with COVID-19 in Wuhan, China: A retrospective cohort study. Lancet. 2020; 395:1054-1062.

6. Goyal P, Choi JJ, Pinheiro LC, et al. Clinical characteristics of Covid-19 in New York city. N Engl J Med. 2020; doi: 10.1056/NEJMc2010419.

7. Ruan QR, Yang K, Wang WX, Jiang LY, Song JX. Clinical predictors of mortality due to COVID-19 based on an analysis of data of 150 patients from Wuhan, China. Intensive Care Med. 2020; doi: 10.1007/s00134-02005991-x.

8. Lau SKP, Lau CCY, Chan KH, Li CPY, Chen H, Jin DY, Chan JFW, Woo PCY, Yuen KY. Delayed induction of proinflammatory cytokines and suppression of innate antiviral response by the novel Middle East respiratory syndrome coronavirus: Implications for pathogenesis and treatment. J Gen Virol. 2013; 94:2679-2690.

9. Beigel JH, Farrar J, Han AM, Hayden FG, Hyer R, de Jong MD, Lochindarat S, Nguyen TK, Nguyen TH, Tran TH, Nicoll A. Avian influenza A (H5N1) infection in humans. N Engl J Med. 2005; 353:1374-1385.

10. National Health Commission of People's Republic of China. http://www.nhc.gov.cn/wjw/index.shtml (accessed March 08, 2020). (in Chinese).

11. Huang CL, Wang YM, Li XW, Ren LL, Zhao JP, Hu Y. Clinical features of patients infected with 2019 novel coronavirus in Wuhan, China. Lancet. 2020; 395:497-506.

12. Sun D, Li H, Lu XX, Xiao H, Ren J, Zhang FR, Liu ZS. Clinical features of severe pediatric patients with coronavirus disease 2019 in Wuhan: A single center's observational study. World J Pediatr. 2020; doi: 10.1007/ s12519-020-00354-4.

13. Wang Z, Yang B, Li Q, Wen L, Zhang R. Clinical features of 69 cases with coronavirus disease 2019 in Wuhan, China. Clin Infect Dis. 2020; pii: ciaa272. doi: 10.1093/ cid/ciaa272.

14. Chen X, Zhao B, Qu Y, Chen Y, Xiong J, Feng Y, Men D, Huang Q, Liu Y, Yang B, Ding J, Li F. Detectable serum SARS-CoV-2 viral load (RNAaemia) is closely correlated with drastically elevated interleukin 6 (IL-6) level in critically ill COVID-19 patients. Clin Infect Dis. 2020; pii: ciaa449. doi: 10.1093/cid/ciaa449.

15. Yang Y, Shen C, Li J, et al. Exuberant elevation of IP-10, MCP-3 and IL-1 ra during SARS-CoV-2 infection is associated with disease severity and fatal outcome. medRxiv. 2020; doi: https://doi. org/10.1101/2020.03.02.20029975

16. Velazquez-Salinas L, Verdugo-Rodriguez A, Rodriguez LL. The role of interleukin 6 during viral infections. Front Microbiol. 2019; 10:1057.

17. McGonagle D, Sharif K, O'Regan A. The role of cytokines including interleukin-6 in COVID-19 induced pneumonia and macrophage activation syndrome- 
like disease. Autoimmun Rev. 2020; doi: 10.1016 j.autrev.2020.102537.

18. Xu Z, Shi L, Wang YJ, et al. Pathological findings of COVID-19 associated with acute respiratory distress syndrome. Lancet Respir Med. 2020; 8:420-422.

19. Schulert GS, Grom AA. Pathogenesis of macrophage activation syndrome and potential for cytokine-directed therapies. Annu Rev Med. 2015; 66:145-159.
Received March 23, 2020; Revised April 22, 2020; Accepted April 27, 2020.

\section{*Address correspondence to:}

Jing Dong, Department of Rheumatology and Clinical Immunology, The Affiliated Hospital of Qingdao University, 16 Jiangsu Road, Shinan District, Qingdao, Shandong, China. E-mail: dongjing@qduhospital.cn 